\title{
Trial Registration for Public Trust: Making the Case for Medical Devices
}

\author{
Ida Sim, MD, PhD ${ }^{1,2}$ \\ 'Division of General Internal Medicine, Department of Medicine, University of California, San Francisco, 400 Parnassus Ave., Rm. A-405, San \\ Francisco, CA 94143-0320, USA; ${ }^{2}$ Center for Clinical and Translational Informatics, University of California, San Francisco, San Francisco, CA \\ 94143-0320, USA.
}

Recently, several pharmaceutical companies have been shown to have withheld negative clinical trial results from the public. These incidents have resulted in a concerted global effort to register all trials at inception, so that all subsequent results can be tracked regardless of whether they are positive or negative. These trial registration policies have been driven in large part by concern about the pharmaceutical sector. The medical device industry is much smaller, and different from the pharmaceutical industry in some fundamental ways. This paper examines the issues surrounding registration of device trials and argues that these differences with pharmaceutical should not exempt device trials from registration.

KEY WORDS: medical device industry; trial registration policies; public trust.

J Gen Intern Med 23(Suppl 1):64-8

DOI: $10.1007 / \mathrm{s} 11606-007-0300-7$

(c) Society of General Internal Medicine 2007

\section{INTRODUCTION}

When most clinicians and patients think of the biomedical industry, it is "Big Pharma" that comes to mind-large multinational pharmaceutical companies that make and sell prescription drugs. The pharmaceutical industry's annual revenues, $\$ 600$ billion worldwide, are triple those of medical devices. The pharmaceutical industry has a considerable presence in the media, and also on Capitol Hill, where the Pharmaceutical Research and Manufacturers of America (PhRMA) is widely considered to be 1 of the most powerful lobbying organizations in the United States. In contrast, its fellow industry sectors, biotechnology and medical devices, have a lower profile in public and in the professional medical world.

One consequence of this differential attention toward the pharmaceutical industry is that public policy affecting the whole biomedical industry may sometimes be disproportionately driven by the features and concerns of the pharmaceutical sector. For example, several pharmaceutical companies have recently been shown to have withheld clinical trial results that were unfavorable to their commercial interests. GlaxoSmithKline (GSK) withheld data on adolescent suicide ideation with paroxetine (Paxil). ${ }^{1}$ Merck withheld data on cardiovascular risk from rofecoxib (Vioxx). ${ }^{2}$ The legal settlement in August 2004 between New York Attorney General Eliot Spitzer and
GSK over the Paxil scandal reflected the seriousness of withholding trial data, and the International Committee of Medical Journal Editors (ICMJE) followed up with a bold move to require that trials must be registered at inception for the results to be considered for publication. ${ }^{3}$ In the last 2 years, a flurry of state and national legislation have been introduced calling for mandatory clinical trial registration, and the World Health Organization (WHO) is establishing international norms and standards to coordinate the global effort to restore public trust in clinical trials through trial registration.

Because the inciting scandals were originally from the pharmaceutical industry, a perception has been that the problem (withheld data) and the solution (mandatory trial registration) apply only to pharmaceutical trials. The device industry argues that fundamental differences between the pharmaceutical and device industries should be reflected in a differentiated regulatory approach. ${ }^{4,5}$ It is thus worth reviewing the differences between the pharmaceutical and device industries, and to consider whether device trials should be exempt from or fall under different trial registration policies than pharmaceutical trials.

\section{DIFFERENCES BETWEEN THE MEDICAL DEVICE AND PHARMACEUTICAL INDUSTRIES}

The medical device and pharmaceutical industries differ in many respects, including size, degree of consolidation, product development cycle, product lifecycle, and their scientific, clinical, and regulatory environments (Table 1). These differences, while true today, are in flux as the biomedical products industry is changing. Drug-device hybrid products such as drug-eluting stents are blurring the line between devices and pharmaceuticals, pharmaceutical firms are increasingly merging with biotechnology firms in an effort to bolster product pipelines, ${ }^{6}$ and the underlying model of pharmaceutical industry innovation appears to be shifting away from blockbuster drugs toward an as-yet-undefined model. ${ }^{7}$

The pharmaceutical industry is a mature, global industry that is comprised primarily of large multinationals. The device industry is younger, less global, and comprised of a few large companies and many more small- to medium-sized companies. The pharmaceutical industry relies on blockbuster drugs (defined as over $\$ 1$ billion in global annual revenues) for profits. ${ }^{7}$ Devices are often designed and targeted for markets of less than $\$ 100$ million in annual revenue. The pharmaceutical industry often speaks through PhRMA, their American trade association, as well as the International Federation of 
Table 1. Summary Differences Between the Pharmaceutical and Device Industries

\begin{tabular}{|c|c|}
\hline Devices industry & Pharmaceutical industry \\
\hline \multicolumn{2}{|l|}{ Industry characteristics } \\
\hline $\begin{array}{l}\text { Annual global revenue US\$200 } \\
\text { billion }\end{array}$ & $\begin{array}{l}\text { Annual global revenue } \\
\sim \text { US\$600 billion }\end{array}$ \\
\hline $\begin{array}{l}\text { Comprised mostly of small- to } \\
\text { medium-sized companies }\end{array}$ & $\begin{array}{l}\text { Comprised primarily of large } \\
\text { multinationals }\end{array}$ \\
\hline Younger, more localized industry & Mature global industry \\
\hline $\begin{array}{l}\text { Represented by AdvaMed, } \\
\text { MEDEC, EucoMed }\end{array}$ & $\begin{array}{l}\text { Represented by PhRMA*, } \\
\text { IFPMA } \dagger\end{array}$ \\
\hline \multicolumn{2}{|l|}{ Products } \\
\hline $\begin{array}{l}\text { Draws upon mechanical, } \\
\text { electrical, materials, and } \\
\text { bio-engineering, now } \\
\text { expanding to biotechnology, etc. }\end{array}$ & $\begin{array}{l}\text { Traditionally centered on } \\
\text { pharmacology and chemistry, } \\
\text { now expanding to } \\
\text { biotechnology, etc. }\end{array}$ \\
\hline $\begin{array}{l}\text { Mechanical or electrical devices, } \\
\text { or both, to replace or augment } \\
\text { body parts and/or function } \\
\text { with more localized effects }\end{array}$ & $\begin{array}{l}\text { Stable, unchanging molecular } \\
\text { entities with systemic effects }\end{array}$ \\
\hline \multicolumn{2}{|l|}{ Product development } \\
\hline $\begin{array}{l}\text { Short iterative cycles each lasting } \\
\text { months, to demonstrate safety, } \\
\text { and effectiveness }\end{array}$ & $\begin{array}{l}\text { Years of clinical trials to } \\
\text { demonstrate safety and efficacy }\end{array}$ \\
\hline $\begin{array}{l}\text { Continuous incremental } \\
\text { improvements introduced } \\
\text { during product evaluation }\end{array}$ & $\begin{array}{l}\text { Product essentially unchanged } \\
\text { during evaluation }\end{array}$ \\
\hline $\begin{array}{l}\text { Annual revenue target usually } \\
<\$ 100 \text { million }\end{array}$ & $\begin{array}{l}\text { Blockbuster annual revenue } \\
\text { target }>\$ 1 \text { billion }\end{array}$ \\
\hline $\begin{array}{l}\text { Investment recovery period as } \\
\text { short as } 18 \text { months }\end{array}$ & Years of market exclusivity \\
\hline $\begin{array}{l}\text { Less reliance on publications of } \\
\text { clinical trial results for marketing }\end{array}$ & $\begin{array}{l}\text { Marketing approach relies more } \\
\text { heavily on clinical trials and } \\
\text { publications }\end{array}$ \\
\hline
\end{tabular}

*Pharmaceutical Research and Manufacturers of America

$\dagger$ International Federation of Pharmaceutical Manufacturers \& Associations

Pharmaceutical Manufacturers \& Associations (IFPMA). The device industry is represented by AdvaMed in the United States, MEDEC in Canada, and Eucomed in Europe.

In pharmaceuticals, the traditional development and innovation process centers on using pharmacology and chemistry to develop a drug - a stable, unchanging molecular entitythat is almost always patented before it undergoes clinical testing. Product development involves years of trials to demonstrate and prove quality, safety, and efficacy for broad populations. Once the product is approved, the company generally has years of market exclusivity in which to recoup costs and generate profits. In contrast, the development and innovation process for devices draws on a wide range of disciplines and technologies, including electrical, mechanical, chemical, and bio-engineering. Patents provide less protection than for pharmaceuticals, because simple design or engineering changes can circumvent a patent. Product development occurs in short iterative cycles each lasting months, with incremental improvements being continuously introduced even during product evaluation. Once on the market, the investment recovery period is comparatively shorter, as little as 18 months in contrast to years for pharmaceuticals.

The regulatory framework for pharmaceuticals and devices also differ substantially. The FDA approves new molecular entities only if clinical data are submitted that demonstrate safety and efficacy. For devices, FDA approval is based on the device's risk classification and may not require any clinical data at all. ${ }^{8}$ Class I devices (e.g., bandages, bedpans) are lowrisk and are governed by "general controls" on packaging, labeling, and the like. Class II devices are moderate-risk (e.g., infusion pumps) and are governed by "special controls" such as mandatory performance standards and requirements for post-market surveillance. Class III devices are high-risk devices (e.g., heart valves) or devices that are not "substantially equivalent" to a similar device already on the market. Class III devices require premarket approval through the submission of clinical and nonclinical data (e.g., design and manufacturing data) to demonstrate safety and effectiveness, but not necessarily efficacy. Overall, the regulatory requirements for demonstrating clinical benefit are less stringent for devices than for pharmaceuticals.

Another distinguishing feature between the device and pharmaceutical industries is that devices are more often developed in closer collaboration with clinicians and are then marketed to them with less reliance on published results of clinical trials. This marketing approach has allowed smaller companies to succeed even if they lack the ability to run large randomized trials. However, as payers increasingly look for evidence of efficacy to support coverage decisions, the demand for clinical trials on devices is increasing, ${ }^{9}$ and therefore also the relevance of trial registration to the device industry. ${ }^{5}$

\section{TRIAL REGISTRATION}

The idea of trial registration, mired for decades in academic backwaters, finally became "irresistible" ${ }^{10}$ as the public realized the harm from withholding negative clinical trial results. To prevent such selective reporting of results, all trials worldwide should be publicly registered and uniquely identified in open access registers, such that all results, whether "good" or "bad," can be tracked down. ${ }^{11}$ Trial registration is a necessary prerequisite to ensuring an unbiased evidence base for patient care and public policy. ${ }^{12}$

Recognizing the need for global coordination of trial registration, the World Health Organization launched the International Clinical Trials Registry Platform in $2005^{13}$ to establish a coordinated network of international trial registers. This network is now operational and includes national and international trial registers. Each participating register offers open access to a standard set of 20 trial-registration data items describing a trial's basic design (Table 2). Trials are to be registered by the Responsible Registrant (either the Principal Investigator or the Primary Sponsor ${ }^{14}$ ) and the trial should be registered once and only once in the network. The WHO Registry Platform is also developing norms and standards for a minimum set of results to be reported for all registered trials.

\section{INDUSTRY CONCERNS REGARDING TRIAL REGISTRATION}

Industry voiced 2 major concerns regarding trial registration. The first concern was whether early trials, commonly known as Phase I trials, should be registered. The second was whether trial registration information should be fully disclosed at the time of registration. Both concerns reflect the tension between protecting competitive advantage for industry and the scientific and ethical responsibilities to trial participants and public health. 
Table 2. World Health Organization Trial Registration Data Set

\begin{tabular}{ll}
\hline \hline & Item \\
\hline 1. & Primary Register and Trial ID \# \\
2. & $\begin{array}{c}\text { Date of Registration in Primary } \\
\text { Register }\end{array}$ \\
3. Secondary ID\#s
\end{tabular}

4. Source(s) of Monetary or Material Support

5. Primary Sponsor

6. Secondary Sponsor(s)

7. Contact for Public Queries

8. Contact for Scientific Queries

9. Public Title

10. Scientific Title

11. Countries of

Recruitment

12. Health Condition(s) or Problem

(s) Studied

13. Intervention(s)

14. Key Inclusion and Exclusion Criteria

15. Study Type

16. Date of First Enrollment

17. Target Sample Size

18. Recruitment Status

19. Primary Outcome(s)

20. Secondary Outcomes
Definition/Explanation

Name of trial register in which this trial was first registered (the trial's "Primary Register"), and the unique ID number assigned by the Primary Register.

Date when trial was officially registered in the Primary Register YYYY/MM/DD.

Other identifying numbers and issuing authorities besides the Primary Register, if any. This includes the sponsor name and sponsor-issued trial number (e.g., protocol number) if available, and other trial registers that have issued an ID number to this trial.

Major source(s) of monetary or material support for the trial (e.g., funding agency, foundation, company).

The individual, organisation, group, or other legal person taking on responsibility for securing the arrangements to initiate and/or manage a study (including arrangements to ensure that the design of the study meets appropriate standards and to ensure appropriate conduct and reporting). The primary sponsor is normally the main applicant for regulatory authorization to begin the study. It may or may not be the main funder.

Additional individuals, organisations or other legal persons, if any, that have agreed with the primary sponsor to take on responsibilities of sponsorship.

Email address, telephone number, or address of the contact who will respond to general queries, including information about current recruitment status

Email address, telephone number, or postal address, and affiliation of the person to contact for scientific inquiries about the trial (e.g., principal investigator, medical director employed by the sponsor). For a multicenter study, the contact should be for the lead principal investigator or overall scientific director.

Title intended for the lay public in easily understood language.

Scientific title of the study as it appears in the protocol submitted for funding and ethical review, including the trial acronym if available.

The countries from which participants will be, are intended to be, or have been recruited

Primary health condition(s) or problem(s) studied (e.g., depression, breast cancer, medication error).

The specific name of the intervention(s) and the comparator/control(s) being studied. The International NonProprietary Name should be used if possible (not brand/trade names). For an unregistered drug, the generic name, chemical name, or company serial number is acceptable. The control intervention(s) is/are the interventions against which the study intervention is evaluated (e.g., placebo, no treatment, active control). If an active control is used, the name(s) of that intervention, or "placebo" or "no treatment" should be provided as applicable. For each intervention, other intervention details should also be described as applicable (dose, duration, mode of administration, etc)

Inclusion and exclusion criteria for participant selection, including age and sex.

A single arm study is one in which all participants are given the same intervention. Trials in which participants are assigned to receive 1 of 2 or more interventions are NOT single arm studies.Crossover trials are NOT single arm studies. A trial is "randomized" if participants are/were assigned to intervention groups using a method based on chance (e.g., random number table, random computer-generated sequence, minimization).

Anticipated or actual date of enrollment of the first participant (YYYY/MM).

Number of participants that this trial plans to or had planned to enroll

Recruitment status of this trial (Pending, Active, Temporary halt, Closed)

Outcomes are events, variables, or experiences that trial investigators measure because it is believed that they may be influenced by the intervention. The Primary Outcome should be the outcome used in sample size calculations, or the main outcome(s) used to determine the effects of the intervention(s).

Secondary outcomes are events, variables, or experiences of secondary interest or that are measured at timepoints of secondary interest. A secondary outcome may involve the same event, variable, or experience as the primary outcome, but measured at time points other than those of primary interest (e.g., primary outcome: all-cause mortality at 5 years; secondary outcome: all-cause mortality at 1 year, 3 years), or may involve a different event, variable, or experience altogether (e.g., primary outcome: all-cause mortality at 5 years; secondary outcome: hospitalization rate at 5 years).

Adapted from the WHO International Clinical Trials Registry Platform, http://www.who.int/ictrp/data_set/en/indexl.html.

\section{Registration of Early Phase Trials}

The pharmaceutical industry has argued against the registration of early-phase studies because these "exploratory" studies are used only "to plan the conduct of confirmatory clinical trials". ${ }^{15}$ The industry claims that these non-hypothesistesting trials "do not generate data which are used to support clinical claims or recommendations for use" and therefore offer little evidentiary value to the scientific and clinical communities. They further claim that registration of early-phase trials would impede innovation because public release of the 20 registration data items would unfairly provide their competitors with development plans and would result in the loss of competitive advantage. In addition to the arguments advanced by the pharmaceutical industry, the device industry also claims that early-phase studies of devices are particularly non-informative for public health because devices undergo such frequent, incremental improvements that the devices tested in early-phase studies are often quite dissimilar to the marketed product ${ }^{4}$. This situation differs from typical drug development where the compound is essentially unchanged from early- to late-phase testing. 
These concerns deserved serious consideration along with the concerns of other stakeholders in clinical trials like the Ottawa Group ${ }^{16}$ that advocated full and complete disclosure of detailed trial informtion. Therefore, WHO convened a safeharbor consultation held in Geneva in 2005, with representatives from the pharmaceutical, device, and biotechnology industries; patient and consumer groups; medical journal editors; ethicists; regulatory officials; and scientists to explore these concerns and to define a common international approach to trial registration. WHO concluded that the claimed threats to proprietary and commercial interests were unconvincing and did not outweigh the benefits of early phase trial registration. ${ }^{16}$ These benefits include the provision of design information that can inform the planning of new trials and help reduce unnecessary duplication. Although the ICMJE originally required the registration of only late-phase "clinically directive" trials, they have since revised their position to require the registration of early- as well as late-phase studies. ${ }^{17}$

The implementation of early-phase trial registration is, however, undoubtedly challenging. It is unknown how many early-phase trials there are-probably thousands worldwide each enrolling small numbers of participants. It is not yet clear that the 20-item data set, originally designed for late-phase studies, is fully appropriate for early studies, or for device studies in particular. It must also be acknowledged that latephase randomized studies are more likely to yield "clinically directive" evidence than early-phase studies so that practically speaking, registration policies might focus on late-phase, "non-exploratory" or "hypothesis-testing" trials. Furthermore, there exist methodological concerns about the appropriateness of standard clinical trial designs for the very iterative nature of early-phase device testing. ${ }^{9}$ It may be reasonable to focus initial efforts in registration of early-phase trials to the pharmaceutical rather than the devices industry.

\section{Disclosure Timing of the Registration Data Set}

The other major concern over trial registration officially voiced by both the pharmaceutical and devices industry was the timing of public access to 5 commercially "sensitive" registration data items ${ }^{4,18}$ : (1) official scientific title of the study; (2) intervention name(s); (3) target sample size; (4) primary outcome; and (5) key secondary outcomes. To protect competitive advantage, the industries proposed withholding these 5 data items from public disclosure until the sponsor (i.e., the company) determines that "the information is no longer commercially sensitive". ${ }^{18}$ This issue of disclosure timing was also addressed at the safe-harbor consultation that the WHO held in Geneva in 2005. Again, WHO found the claim that full and immediate disclosure of registration data would threaten the industry's competitive health to be unconvincing, and that these threats would not in any case outweigh the benefits to the public good of immediate disclosure. ${ }^{16}$

In this matter of disclosure timing of registration information, none of the differences between the pharmaceutical and device industries were material. AdvaMed argued that because the device industry has less patent protection, the "forced disclosure of intellectual property" from trial registration will be particularly injurious. ${ }^{4}$ However, as in the pharmaceutical industry, the 5 "sensitive" items are often already available to competitors through fee-based commercial intelligence services or through patient consent forms that can be obtained publicly. If competitors within the industry already have access to the 5 "sensitive" items, then there is no case for limiting public access to them.

\section{ETHICS AND POLICY ISSUES IN DEVICE TRIAL REPORTING}

Experimenting on human beings is a serious matter. In enunciating the core ethical principles on human research, the Declaration of Helsinki states explicitly that "the design of all studies should be publicly available". ${ }^{19}$ Trial registration aims to meet this ethical duty by making public the design and existence of all trials. No commercial sector can claim exemption from this duty. This duty extends even to small earlyphase trials as every trial participant, whether as 1 of 6 or 6,000 participants, deserves the same considerations under the Declaration of Helsinki.

There are also no ethical grounds for the device and pharmaceutical industries' claims of exemption on the basis that trial registration would threaten competition or innovation. The Nuremburg Code states unequivocally that human experiments "should be such as to yield fruitful results for the good of society". ${ }^{20}$ Study participants volunteer for trials expecting to improve medical knowledge in general, not to increase a company's profit. Moreover, other industries have experienced greater, not less, innovation with open disclosure $^{21}$ so it could be argued that full and open registration might contribute to the good of industry and society together.

The most recently proposed U.S. Congressional legislation on this topic, "Enhancing Drug Safety and Innovation Act of 2007," affirms the broad duty to register at least late-phase trials. The legislation requires registration of all trials except "exploratory" trials "intended solely to assess safety or solely to evaluate pharmacokinetics" or "to determine the feasibility of a device." The House of Representatives' version of the bill (WaxmanMarkey) specifically includes the device industry in its trial registration requirements, ${ }^{22}$ whereas the corresponding Senate bill (Enzi-Kennedy) does not. ${ }^{23}$ These bills also do not specifically require public disclosure of all registration data at the time of registration. Registration data must be submitted not later than 14 days after the first patient is enrolled, but the bills appear to leave open the possibility that industry, for example, could have the choice to withhold some data items from public disclosure. One notable point about the bills is their introduction of penalties for failure to register. This is crucial as voluntary trial registration has been shown to be incomplete. ${ }^{24,25}$

\section{CONCLUSION}

The Paxil and Vioxx scandals, among others, were only sentinel events that revealed a structural flaw in the clinical trials enterprise that allows for the mis-representation of trial results from any sector, whether pharmaceutical or devices, commercial or academic. It is this systematic lack of accountability to the "good of society" that is eroding public trust in clinical trials. Whereas a global consensus has now solidified on the importance of trial registration to counteract this erosion, the practicalities of trial registration remain daunting. Industry support and participation is a must, as an increasing proportion of trials are being conducted in the private sector. ${ }^{26}$ 
A balancing of ethical duties of human experimentation and the needs of particular industries will be needed if we are to continue strengthening the overall integrity of clinical trials research.

Disclosure: Ida Sim served as the founding Project Coordinator of the World Health Organization's International Clinical Trials Registry Platform from August 2005 to December 2006. The views in this article are those of Ida Sim and do not necessarily reflect the views of WHO or UCSF.

Corresponding Author: Ida Sim, MD, PhD; Center for Clinical and Translational Informatics, University of California, San Francisco, San Francisco, CA 94143-0320, USA (e-mail: ida.sim@ucsf.edu).

\section{REFERENCES}

1. People of the State of New York vs GlaxoSmithKline. Filed June 2, 2004

2. Curfman GD, Morrissey S, Drazen JM. Expression of concern: Bombardier et al., Comparison of upper gastrointestinal toxicity of rofecoxib and naproxen in patients with rheumatoid arthritis. N Engl J Med. 2000;343:1520-8. N Engl J Med 2005;353(26):2813-4.

3. DeAngelis CD, Drazen JM, Frizelle FA, et al. Clinical trial registration: a statement from the international committee of medical journal editors. JAMA. 2004;292(11):1363-4.

4. AdvaMed Response to the World Health Organization (WHO) International Clinical Trials Registry Platform (ICTRP) Request for Comments on Delayed Disclosure. March 31, 2006.

5. Popp RL, Lorell BH, Stone GW, Laskey W, Smith JJ, Kaplan AV. An outline for public registration of clinical trials evaluating medical devices. J Am Coll Cardiol. 2006;47(8):1518-21.

6. Profitless prosperity. The Economist, April 20, 2006.

7. Cutler D. The demise of the blockbuster? N Engl J Med. 2007;356 (13): 1292-3.

8. Maisel WH. Medical device regulation: an introduction for the practicing physician. Ann Intern Med. 2004;140(4):296-302.

9. Califf RM. Evaluation of diagnostic imaging technologies and therapeutics devices: better information for better decisions: proceedings of a multidisciplinary workshop. Am Heart J. 2006;152(1):50-8.
10. Rennie D. Trial registration: a great idea switches from ignored to irresistible. JAMA. 2004;292(11):1359-62.

11. Dickersin K, Rennie D. Registering clinical trials. JAMA. 2003;290 (4):516-23.

12. Krleza-Jeric K, Chan AW, Dickersin K, Sim I, Grimshaw J, Gluud C. Principles for international registration of protocol information and results from human trials of health related interventions: Ottawa statement (part 1). BMJ. 2005;330(7497):956-8.

13. World Health Organization. International Clinical Trials Registry Platform. Available at: http://www.who.int/ictrp/. Accessed March 29, 2007.

14. World Health Organization. International Clinical Trials Registry Platform Glossary. Available at: http://www.who.int/ictrp/glossary/en/ index.html. Accessed March 29, 2007.

15. IFPMA Comments to ICTRP Consultation on Delayed Disclosure. January 25, 2006. Available at: http://www.who.int/ictrp/3005_IFPMA_ 25Jan06.pdf. Accessed March 29, 2007.

16. Sim I, Chan AW, Gülmezoglu AM, Evans T, Pang T. Clinical trial registration: transparency is the watchword. Lancet. 2006;367 (9523): 1631-3.

17. Laine C, Horton $\mathbf{R}$, Deangelis $\mathbf{C D}$, et al. Clinical trial registration: Looking back and moving ahead. N Engl J Med. 2007;356(26):2734-6.

18. Lassman S. RE: PhRMA comments on international clinical trials registry platform (ICTRP) disclosure timing. January 25, 2006. Available at: http://www.who.int/ictrp/3010_PhRMA_25Jan06.pdf. Accessed March 29, 2007.

19. World Medical Association. Declaration of Helsinki. 9 Oct 2004. Available at: http://www.wma.net/e/policy/b3.htm. Accessed March 29, 2007.

20. Nuremberg Code. Trials of war criminals before the Nuremberg military tribunals under control council law No. 10. In: U.S. Government Printing Office; Washington, D.C; 1949:181-2.

21. Palmisano S. The information puzzle. Newsweek, December 2, 2005.

22. Enhancing Drug Safety and Innovation Act, H.R. 1561, 110th Congress, 1st Session (2007).

23. Enhancing Drug Safety and Innovation Act, S. 484, 110th Congress, 1st Session (2007).

24. Derbis J, Toigo T, Woods J, Evelyn B, Banks D. FDAMA Section 113: Information program on clinical trials for serious or life threatening diseases: update on implementation. In: 9th Annual FDA Science Forum. Washington, D.C; 2003.

25. Zarin DA, Tse T, Ide NC. Trial registration at ClinicalTrials.gov between May and October 2005. N Engl J Med. 2005;353(26):2779-87.

26. Patsopoulos NA, Ioannidis JP, Analatos AA. Origin and funding of the most frequently cited papers in medicine: database analysis. BMJ. 2006;332(7549):1061-4. 